\title{
ASPECTOS ECONÓMICO-CONSTITUCIONALES DE LOS DESC Y LOS DERECHOS CIVILES Y POLÍTICOS EN MÉXICO
}

\author{
Alejandra Verónica Zúñiga Ortega
}

doi: 10.18543/ed-63(2)-2015pp283-293

Sumario: 1. Derecho Constitucional ECONÓMico meXicano. 2. DESC VERSUS DERECHOS CIVILES Y POLÍtICOS. 3. COLOFÓN. 4. FUENTES DE CONSULTA.

\section{DERECHO CONSTITUCIONAL ECONÓMICO MEXICANO}

Cuando se habla de derecho constitucional económico, se alude al «conjunto de normas y principios que, recogidos en la Constitución, tienen efecto patrimonial sea para el Estado, para los individuos o para ambos $\rangle^{1}$, o lo que también es conocido como como Constitución económica, es decir, el «...marco jurídico fundamental de la estructura y funcionamiento de la actividad económica...» ${ }^{2}, o$ bien, en palabras de Bidart, «...conjunto de normas, principios y valores que, una vez incorporados a la Constitución formal, guardan relación con la economía y son aplicables a la actividad y a las relaciones económicas financieras $\rangle^{3}$.

${ }^{1}$ VizCAÍNO LÓPEZ, M. T. «El derecho constitucional económico y sus principios en el estado mexicano», IUS Revista jurídica, Universidad Latina de América, disponible en: $<$ http://www.unla.mx/iusunla20/reflexion/derecho \%20constitucional \%20economico $\% 20 y \% 20$ sus \%20prin.htm. >, fecha de consulta 06 de octubre de 2015.

2 Torres del Moral,A., Principios de Derecho constitucional español, Publicaciones UCM, Madrid, 2010, 6. ${ }^{\text {a }}$ ed., p. 596.

3 Bidart Campos, G., «Constitución económica», Diccionario Iberoamericano de Derechos Humanos y Fundamentales, Universidad de Alcalá, 2002, p. 1144. 
Las Constituciones de algunos países destinan uno o varios capítulos específicos a la parte económica que rige la función estatal en esta materia, prueba de ello es Colombia ${ }^{4}$, Uruguay ${ }^{5}$ y Brasil ${ }^{6}$, por mencionar solo algunos.

En la Constitución Política de los Estados Unidos Mexicanos no existe un apartado concreto sobre el régimen económico, de finanzas o de hacienda pública, como prefiera calificarse, lo que se tiene es una serie de artículos que regulan:

La rectoría del desarrollo nacional bajo los criterios de fortalecimiento de la Soberanía y régimen democrático, competitividad, fomento económico y empleo, así como, justa distribución del ingreso y la riqueza, con el objeto de permitir el pleno ejercicio de la libertad y la dignidad de los individuos, grupos y clases sociales. Y la economía mixta, es decir, la participación de los sectores, público, privado y social en el desarrollo económico (artículo 25).

La planeación democrática, por consulta popular, del desarrollo nacional mediante el plan nacional de desarrollo coordinado por el Ejecutivo Federal (artículo 26).

La propiedad originaria, es decir, el dominio que la Nación ejerce sobre su territorio, que le corresponde «originariamente», mismo que puede ser transmitido a los particulares, constituyendo la propiedad privada. (artículo 27).

Los principios antimonopolios y los postulados del sistema bancario y financiero (artículo 28).

Aunado a este sector de artículos, las funciones económicas del Estado, llevadas a cabo por el Poder Ejecutivo en los tres niveles de gobierno, federal, estatal y municipal, se manifiestan en las siguientes actividades ${ }^{7}$ :

a) Obtención de recursos económicos indispensables para un óptimo ejercicio del aparato estatal, a través de la aplicación de la Ley de Ingresos de la Federación y demás ordenamientos de carácter fiscal.

b) Administración de los recursos financieros por medio del ejercicio del Presupuesto de Egresos de la Federación.

c) Desarrollo de políticas públicas acordes con el Plan Nacional de Desarrollo y programas sectoriales.

Diseño e implementación de ordenamientos legales que brinden seguridad jurídica a inversionistas.

4 Título XII «Del régimen económico y de la hacienda pública», capítulos I-VI, artículos 332-373.

5 Sección XIV «De la hacienda pública», capítulos I-VI, artículos 214-232.

6 Título VI «De la tributación y del presupuesto», capítulos I II, artículos 145-169 y Título VII «Del orden económico y financiero», capítulos I-IV, artículos 170-192.

7 VALERo Flores, C. N., El capítulo económico de la Constitución y el desarrollo de México, Cámara de Diputados, Serie verde, México, 2008, p. 22 
Estas funciones, así como las obras y servicios públicos, se encaminan al cumplimiento de los fines comunes de todo Estado, esto es, la auto supervivencia del mismo a través de la satisfacción de las necesidades públicas, la salvaguarda del orden público, el logro del bien común y, en el tema que nos ocupa, el resguardo del ejercicio de los derechos civiles, políticos, económicos, sociales y culturales.

Ahora bien, volviendo al capítulo económico de la Constitución Mexicana, compartiendo la posición de Valero ${ }^{8}$, no sólo los numerales enunciados se relacionan con la parte económica, sino que existen otros que están íntimamente relacionados con la actividad económica estatal, y que se encuentran insertos en los derechos económicos, sociales y culturales, comúnmente llamados DESC, esto es, entre otros:

- El artículo 123 regula lo relativo a los derechos de los trabajadores, en los que se circunscriben la prohibición de trabajo forzado, el derecho a escoger libremente un trabajo, un salario equitativo por trabajo de igual valor, goce de tiempo libre, limitación razonable de las horas laborales, seguridad e higiene en las instalaciones del trabajo, el derecho de afiliarse y fundar sindicatos, el derecho la huelga, así como, el derecho a la seguridad y protección social, en los que se insertan los derechos a «... la no denegación de la cobertura de la seguridad social de manera arbitraria o no razonable y ... la igualdad en el disfrute de la adecuada protección en caso desempleo, enfermedad, vejez o falta de medios de subsistencia en circunstancias que escapan al control de la persona».

- Y el artículo 4. ${ }^{\circ}$, que contempla el derecho a un nivel de vida adecuado, el cual incluye el derecho a la alimentación, al agua, al vestido, a una vivienda digna y la protección contra el hambre ${ }^{9}$, así como, la asistencia y la protección de la familia, vinculadas con los derechos, especialmente de la niñez, de alimentación, salud, educación y sano esparcimiento.

A su vez, a estos artículos de carácter económico, cuyo contenido son derechos sociales, tradicionalmente se les vincula con las obligaciones «de hacer» del Estado y con la «carga presupuestaria» que supone su ejercicio; en consecuencia, son contrapuestos con aquellas obligaciones y efectos económicos «livianos» que devienen de los derechos civiles y políticos. Sin embar-

${ }^{8}$ Ibidem, p. 13.

9 Oficina del Alto Comisionado de las Naciones Unidas para los Derechos Humanos, Preguntas frecuentes sobre los Derechos Económicos, Sociales y Culturales, folleto informativo núm. 33, Ginebra, pp. 11-12, <www.ohchr.org/Documents/Publications/FS33_sp.pdf>, consultado el 28 de octubre de 2015. 
go, dichas obligaciones estatales y el impacto económico que originan tanto los DESC como los derechos civiles y políticos, debieran ser analizadas retomando la esencia de cada derecho en particular, en contraste con los actos materiales o abstenciones que el Estado debe realizar o no, para su pleno ejercicio; sin hacer diferencia entre lo social y lo civil o político. Razonamiento que se pretende a continuación.

\section{DESC VERSUS DERECHOS CIVILES Y POLÍTICOS}

Tradicionalmente se ha cuestionado, diferenciado y categorizado a los derechos civiles y políticos respecto de los DESC, principalmente por el tipo de obligaciones que originan y, en consecuencia, por su carácter económico, es decir, por el impacto en el patrimonio estatal, o lo que es lo mismo, por el apartado de las normas constitucionales económicas que les son inherentes.

Tal como lo evidencian Courtis y Abramovich al fundamentar acerca de la justiciabilidad de los DESC

«...Uno de los argumentos que se repiten para sostener la pretendida distinción de los derechos civiles y políticos con respecto a los derechos económicos, sociales y culturales, radica en el supuesto carácter de obligaciones negativas del primer género de derechos, mientras que los derechos económicos, sociales y culturales implicarían el nacimiento de obligaciones positivas que en la mayoría de los casos deben solventarse con recursos del erario público. De acuerdo a esta posición, las obligaciones negativas se agotarían en un no hacer por parte del Estado: no detener arbitrariamente a las personas, no aplicar penas sin juicio previo, no restringir la libertad de expresión, no violar la correspondencia ni los papeles privados, no interferir con la propiedad privada, etc. Por el contrario, la estructura de los derechos económicos, sociales y culturales se caracterizaría por obligar al Estado a hacer, es decir, a brindar prestaciones positivas: proveer servicios de salud, asegurar la educación, a sostener el patrimonio cultural y artístico de la comunidad. En el primer caso, bastaría con limitar la actividad del Estado, prohibiéndole su actuación en algunas áreas. En el segundo, el Estado debería necesariamente erogar recursos para llevar a cabo las prestaciones positivas que se le exigen $\rangle^{10}$.

${ }^{10}$ Abramovich, V. y Courtis, C., «Hacia la exigibilidad de los derechos económicos, sociales y culturales. Estándares internacionales y criterios de aplicación ante los tribunales locales», p. 2. <http://r.search.yahoo.com/ ylt=AwrTcd8grFhW8LwAyhDv 8wt.; ylu=X3oDMTByMjAxbTBkBHNlYwNzcgRwb3MDNQRjb2xvA2dxMQR2dGlk $A w--/ R V=2 / R E=1448680609 / R O=10 / R U=h t t p \% 3 a \% 2 f \% 2$ fobservatoridesc.org $\% 2 f$ sites \%2fdefault \%2ffiles \%2fExigibilidad_de_los_DESC_-_Abramovich.pdf $/ R K=0 /$ $R S=U s 3$ VekAcGbXXYeN.HHoQaOtxs.I->, consultado el 13 de septiembre de 2015. 
Para el análisis que nos ocupa, la teoría analítica sobre los derechos fundamentales de Robert Alexy ${ }^{11}$ aportará los elementos indispensables para referiremos a la modalidad de obligaciones que generan tanto los derechos civiles y políticos como los DESC, así como el efecto financiero que traen consigo, específicamente en lo relativo al análisis de los derechos a algo, en el ámbito de los derechos frente al Estado, los cuales, divide según su objeto en derechos a acciones negativas o también llamados derechos de defensa y derechos a acciones positivas (que conllevan parcialmente a los llamados derechos a prestaciones).

A su vez los derechos a acciones negativas por parte del Estado los subdivide en tres grupos:

a) Derechos a que el Estado no impida u obstaculice algunas acciones del titular del derecho, es decir, aquellos que no pueden realizarse ya por la presencia de circunstancias que hacen fácticamente imposible realizar la acción, ya por la creación de circunstancias que pueden impedir realizar la acción.

b) El segundo grupo de los derechos a acciones negativas son los derechos a la no afectación de propiedades (vivir y estar sano) y situaciones (inviolabilidad del domicilio).

c) Por cuanto hace al tercer grupo: derechos a la no eliminación de posiciones jurídicas, Alexy alude, como prototipo de posición jurídica al propietario, estableciendo que para que las posiciones jurídicas, y en especial la propiedad, tengan vida jurídica es necesaria la existencia de normas jurídicas que definan la institución de la propiedad.

Mientras que los derechos a acciones positivas los divide en dos grupos: derechos a acciones positivas fácticas o también llamados derechos a prestaciones en sentido estricto y derechos a acciones positivas normativas o derechos a prestaciones en sentido amplio. Clasificando de este modo los derechos a prestaciones. Según nuestro autor, cuando se hace alusión a estos, generalmente se hace referencia a acciones positivas fácticas, eliminando la posibilidad de hablar de prestaciones normativas.

Los derechos cuyo objeto es una acción fáctica sugieren la creación de medios o condiciones materiales, es decir, aportes reales del Estado. Mientras que los derechos a acciones positivas normativas o derechos a prestaciones en sentido amplio son derechos a actos estatales de imposición de normas.

Dada esta teoría, tanto los derechos civiles y políticos como los DESC son derechos a algo, debido a que en ellos se toma como referencia al desti-

11 AlEXY, R., Teoría de los derechos fundamentales, traducido por GARZÓN VALDÉZ, E., Centro de estudios constitucionales, Madrid, 1997. 
natario del derecho, es decir, al Estado o a los particulares. Pero también, estos derechos a algo implican una relación triádica entre el citado destinatario, el portador o titular del derecho y el objeto del derecho que siempre es una acción ${ }^{12}$ y que en el caso que nos ocupa, consiste en acciones negativas y acciones positivas por parte del Estado, es decir, el objeto es común tanto en los derechos civiles y políticos como en los DESC.

A continuación se presenta un cuadro que sintetiza lo anterior.

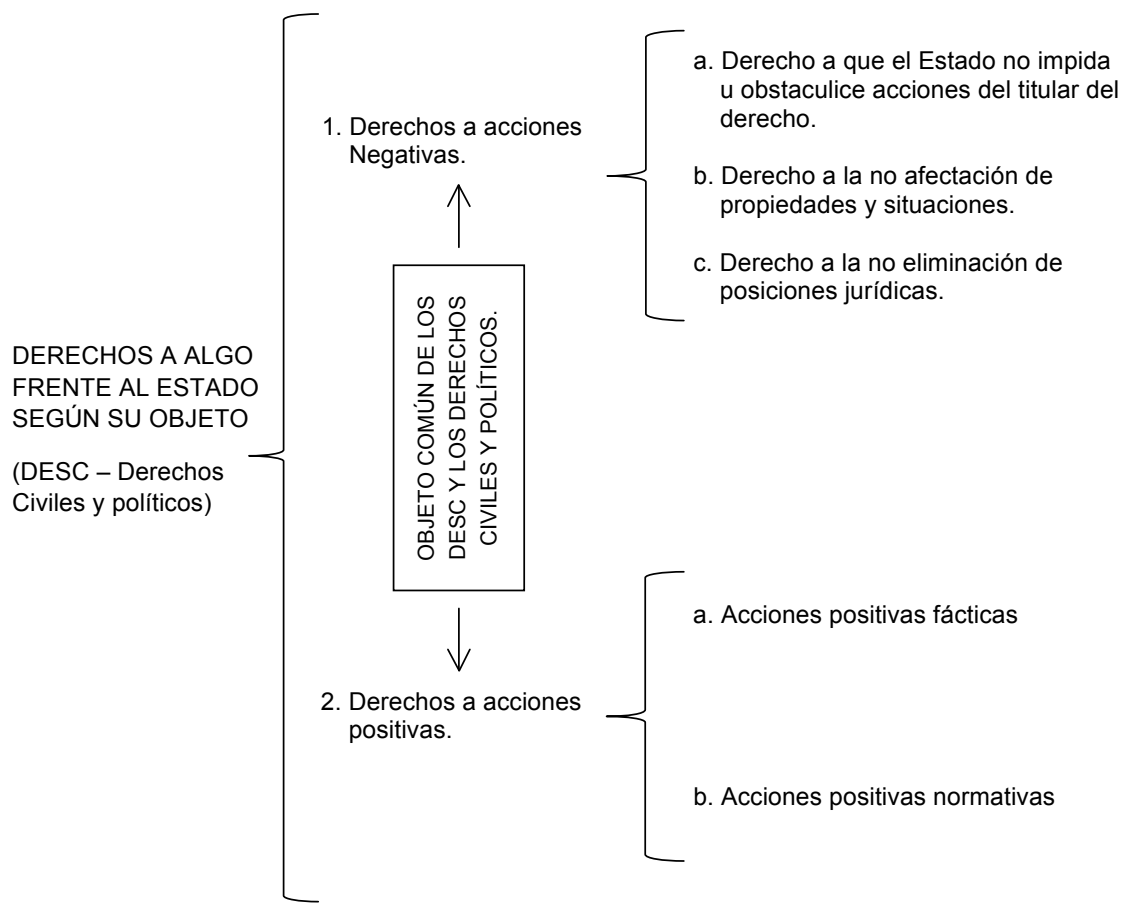

Para evidenciar lo expuesto, piénsese por ejemplo en el derecho a contraer matrimonio, que la Convención Americana Sobre Derechos Humanos reconoce como derecho civil en su artículo 17, apartado 2 y a su vez, determina como deber estatal. Para que dos personas puedan contraer matrimonio, el Estado debe contar con la infraestructura, equipamiento y material de oficina, así como, contratar personal necesario para realizar las funciones propias del Registro Civil, lo que evidentemente trae consigo una erogación económica estatal; en otras palabras, el ejercicio del derecho a contraer ma-

12 Ibidem., pp. 186-210. 
trimonio es entonces un derecho civil cuyo objeto requiere el aporte material estatal (acciones positivas fácticas). No obstante, al mismo tiempo, existe una obligación estatal de no obstaculizar o impedir que se celebre la unión matrimonial, siempre que se cumplan con los requisitos legales establecidos, es decir, el derecho a contraer matrimonio es un derecho civil cuyo objeto supone también acciones negativas.

Frente al derecho a contraer matrimonio considerado como derecho civil, ahora pensemos en el derecho a la educación, incluido en el artículo 26 de la Convención Americana Sobre Derechos Humanos, en el capítulo III intitulado «Derechos económicos, sociales y culturales». Para que el titular del derecho a la educación pueda acceder a él, el Estado edifica inmuebles para el desarrollo de las actividades concernientes a la vida académica y administrativa respectiva, paga sueldo a los profesores y empleados, ofrece cursos de capacitación y actualización al personal docente e implementa programas de diversa índole para mejoramiento de condiciones en el ejercicio del derecho, entre muchas otras más acciones. Lo que visiblemente supone, que este derecho social como derecho a algo, trae consigo la obligación estatal de proporcionar medios materiales (acciones positivas); sin embargo, tal como se observó en el derecho a contraer matrimonio, en el derecho a la educación también existe la obligación estatal de no obstaculizarla, por ejemplo, no puede impedir el ingreso a escuelas a personas con discapacidad o que profesen una religión específica (acciones negativas).

Entonces, desde esta perspectiva, cada uno de los llamados derechos civiles y políticos y los DESC, ambos concebidos como derechos a algo, con acciones positivas y negativas, transitan en una especie de instrumento de nivelación, que determina, según el derecho de que se trate y sin hacer distinciones entre lo civil, político, económico, social y cultural, el grado o la naturaleza de las acciones positivas o negativas a cargo del Estado. Si esto es así, entonces las funciones económicas a las que hicimos referencia anteriormente, se encaminan al ejercicio no sólo de los DESC, sino también de los derechos civiles y políticos.

Los motivos de diferenciación entre los derechos civiles y políticos y los DESC van de lo histórico a la vaguedad del contenido de los DESC, así lo afirma la Oficina del Alto Comisionado de las Naciones Unidas para los Derechos Humanos al establecer que:

«En primer lugar, la distinción se hizo inicialmente por razones históricas. Si bien la Declaración Universal de Derechos Humanos no hacía ninguna distinción entre derechos, ésta apareció en el contexto de la intensificación de las tensiones de la guerra fría entre el Este y el Oeste. Las economías de mercado del Oeste solían hacer más énfasis en los derechos civiles y políticos, en tanto que las economías de planificación centralizada del bloque oriental destacaban la importancia de los derechos económicos, 
sociales y culturales. Eso dio lugar a la negociación y aprobación de los pactos: uno de derechos civiles y políticos y otro de derechos económicos, sociales y culturales. Sin embargo, esa rígida separación se ha abandonado y se ha producido un restablecimiento de la arquitectura original de la Declaración Universal. En los últimos decenios, los tratados de derechos humanos, como la Convención sobre los Derechos del Niño o la Convención sobre los derechos de las personas con discapacidad, han integrado todos los derechos.

En segundo lugar, se considera que los derechos económicos, sociales y culturales requieren un mayor grado de inversión, en tanto que los derechos civiles y políticos requieren que el Estado se abstenga de interferir en las libertades individuales. Es cierto que muchos derechos económicos, sociales y culturales requieren en ocasiones grandes inversiones -tanto financieras como humanas- para garantizar su pleno disfrute. No obstante, los derechos económicos, sociales y culturales también exigen que el Estado se abstenga de interferir en las libertades individuales, como las sindicales o el derecho a elegir el trabajo que se desee. Paralelamente, los derechos civiles y políticos, aunque abarcan libertades individuales, también requieren inversiones para lograr su plena efectividad. Por ejemplo, los derechos civiles y políticos exigen ciertas infraestructuras, como un sistema judicial que funcione, un sistema penitenciario que respete condiciones de vida mínimas para los reclusos, asistencia letrada, elecciones libres e imparciales, etc.

En tercer lugar, los derechos económicos, sociales y culturales en ocasiones se consideran vagos o poco claros por comparación con los derechos civiles y políticos. Si bien no todos los derechos económicos, sociales y culturales están claramente definidos del mismo modo en los tratados de derechos humanos, esto también ocurre con los derechos civiles y políti$\cos \ldots\rangle^{13}$

Así mismo, Nino, al hablar sobre los derechos sociales, afirma que

«Derechos tales como aquéllos a la salud, a la vivienda, a la alimentación, a condiciones dignas de trabajo son incluidos frecuentemente en la categoría de «derechos sociales». De hecho, la etiqueta es equívoca en tanto que conduce a pensar que se sitúan en oposición a los derechos individuales, y esto es así porque son disfrutados por grupos y no por individuos, o porque presuponen la pertenencia a una comunidad como una condición necesaria para su titularidad. En lugar de esto, la concepción liberal de la sociedad ve a estos derechos como una extensión natural de los derechos individuales... Incluso no son diferentes de los derechos a la vida, a la integridad personal y a los demás que se refieren a bienes que son necesarios para la autonomía personal. Generalmente se refieren a condi-

13 Oficina del Alto Comisionado de las Naciones Unidas para los Derechos Humanos, op. cit., pp. 11-12. 
ciones para el goce de esos derechos, además de aquellas condiciones que tradicionalmente han sido tomadas en cuenta» ${ }^{14}$.

Ahora bien, a los DESC, por dotarlos de una imagen prestacional y onerosa en razón de su «exclusividad» de las obligaciones positivas estatales para su óptimo ejercicio, se les encajona en el sentido de generar un solo tipo de obligaciones estatales: obligaciones de hacer.

Como lo observamos, el Estado está obligado a realizar acciones positivas y negativas para que los derechos civiles, políticos, económicos, sociales y culturales tengan relevancia práctica, pero estas acciones se realizan en una especie de combinación en cada uno de los derechos y no encasillándola según sea derecho social o derecho civil y político.

De tal manera, los derechos civiles, políticos, económicos, sociales y culturales son derechos a prestaciones en grados diversos y oscilan entre la abstención estatal y las obligaciones positivas.

Reflexionemos sobre el ejercicio del derecho a la protección de la familia, en éste el Estado no sólo está obligado a acciones positivas, como «conceder especial protección a las madres durante un período de tiempo razonable antes y después del parto» ${ }^{15}$ que implica personal especializado para su atención, material y equipo adecuado, así como, atención médica eficiente y oportuna; sino que también existe la obligación estatal de no negar atención médica y quirúrgica a las mujeres embarazadas. Tratándose de los derechos civiles, en el derecho al reconocimiento de la personalidad jurídica que tiene todo ser humano, una persona transexual a efecto de hacerlo exigible, podría poner en movimiento la maquinaria jurisdiccional, al ser negada un acta de nacimiento por parte del Registro Civil que ostente su sexo psíquico, lo cual también genera acciones estatales positivas.

Así mismo, al atribuírsele el carácter oneroso a los DESC, derivado de las obligaciones positivas a cargo del Estado, se les conciben como derechos «caros» cuyo ejercicio depende de los recursos económicos disponibles. A pesar de ello, los derechos civiles y políticos igualmente requieren de financiamiento público para ser efectivos.

Otra muestra se observa en el ejercicio del derecho al voto. En este el Estado debe poner en movimiento a la estructura electoral que permita al ciudadano emitirlo el día de la jornada, erogando recursos económicos dirigidos a la contratación de personal que capacite a los funcionarios de casilla

${ }^{14}$ Nino, C. S., «Sobre los derechos sociales» en CARBonell, M., et al., Derechos sociales y derechos de las minorias, UNAM, México, 2000, p. 137, <http://biblio.juridicas.unam.mx/libros/libro.htm? $l=1658>$, consultado el 20 de octubre de 2015.

15 Artículo 10, párrafo 2, Pacto Internacional de Derechos Económicos Sociales y Culturales, <http://www.cinu.org.mx/onu/documentos/pidesc.htm>, consultado el 13 de octubre de 2015 . 
o impresión de material electoral, por mencionar sólo algunos casos. Amén de que, concebido así, el artículo 35 constitucional que enmarca los derechos de los ciudadanos mexicanos, entre otros, también formaría parte del derecho constitucional económico que enunciábamos al inicio.

Así las cosas, frente a los derechos civiles, políticos, económicos, sociales y culturales, el Estado se mueve entre la abstención (acción negativa) y las acciones positivas, y es en estas cuando considerados en su globalidad, generan un impacto económico en el Estado.

\section{COLOFÓN}

El derecho constitucional económico se vincula con los DESC y con los derechos civiles y políticos, en primer lugar, porque se fundamentan en el Plan Nacional de Desarrollo, que es el documento de trabajo que regula el presupuesto y programación de la Administración Pública Federal, mismo cuya planeación forma parte de ese conjunto de artículos que conforman el derecho constitucional económico del que hablábamos en un inicio, y que proyecta «hacer de México una sociedad de derechos en donde todos tengan acceso efectivo a los derechos que otorga la Constitución ${ }^{16}$. En segundo lugar, porque este Plan Nacional de Desarrollo, a su vez, se relaciona con una función económica estatal: Desarrollo de políticas públicas y programas sectoriales. Y en tercer lugar, por ese carácter patrimonial, oneroso, de carga económica del Estado, para la efectividad de los mencionados derechos, que no sólo se inclina hacia el ejercicio de los DESC sino también al de los derechos civiles y políticos; propiedad que deriva de su particularidad de ser «derechos a algo», particularmente, por cuanto hace a las acciones positivas estatales, tema central del presente escrito.

\section{FUENTES DE CONSULTA}

Abramovich, V. y Courtis, C., «Hacia la exigibilidad de los derechos económicos, sociales y culturales. Estándares internacionales y criterios de aplicación ante los tribunales locales», <http://r.search.yahoo.com/_ylt=AwrTcd8grFhW8LwAyh Dv8wt.; ylu=X3oDMTByMjAxbTBkBHNlYwNzcgRwb3MDNQRjb2xvA2dxMQ $R 2 d G l k A w--/ R V=2 / R E=1448680609 / R O=10 / R U=h t t p \% 3 a \% 2 f \% 2$ fobservatoridesc.org \%2fsites \%2fdefault \%2ffiles \%2fExigibilidad_de_los_DESC_-_Abramovich.pdf $/ R K=0 / R S=U s 3$ VekAcGbXXYeN.HHoQaOtxs.I->, consultado el 13 de septiembre de 2015.

Alexy, R., Teoría de los derechos fundamentales, traducido por GARZón VAlDÉZ, E., Centro de estudios constitucionales, Madrid, 1997.

$16<$ http://pnd.gob.mx/> 
Bidart Campos, G., «Constitución económica» Diccionario Iberoamericano de Derechos Humanos y Fundamentales, Universidad de Alcalá, 2002.

Nino, C. S., «Sobre los derechos sociales» en Carbonell, Miguel, et al., Derechos sociales y derechos de las minorias, UNAM, México, 2000, pp. 137-143, <http:// biblio.juridicas.unam.mx/libros/libro.htm?l=1658>, consultado el 20 de octubre de 2015.

Oficina del Alto Comisionado de las Naciones Unidas para los Derechos HuMANos, Preguntas frecuentes sobre los Derechos Económicos, Sociales y Culturales, folleto informativo núm. 33, Ginebra, <www.ohchr.org/Documents/Publications/FS33 sp.pdf $>$, consultado el 28 de octubre de 2015.

Torres del Moral, A., Principios de Derecho constitucional español, Publicaciones UCM, Madrid, 2010, 6. ${ }^{\text {a }}$ ed.

VAlero Flores, C. N., El capítulo económico de la Constitución y el desarrollo de México, Cámara de Diputados, Serie verde, México, 2008.

VizCAÍNO LÓPEZ, M. T., «El derecho constitucional económico y sus principios en el estado mexicano, IUS Revista jurídica, Universidad Latina de América, <http:// www.unla.mx/iusunla20/reflexion/derecho \%20constitucional \%20economico $\% 20 y \% 20$ sus \%20prin.htm>, fecha de consulta 06 de octubre de 2015.

TITLE: Economics-constitutional ESCR and civil and political rights in Mexico.

RESUMEN: La vinculación entre el derecho constitucional económico mexicano y los derechos económicos, sociales, culturales, civiles y politicos, se visualiza en el Plan Nacional de Desarrollo, que a su vez se liga con una función económica estatal, asi como, en el carácter patrimonial que comparten los mencionados derechos para su efectividad, atributo que se deriva de la aplicación de la teoría de los derechos fundamentales de Robert Alexy, concretamente desde la perspectiva de los derechos a algo frente al Estado según su objeto, tema central del presente escrito.

PALABRAS CLAVE: Constitución, derechos y económico.

ABSTRACT: The link between the Mexican economic constitutional law and economic, social, cultural, civil and political rights, is displayed in the National Development Plan, which in turn is linked with state economic function and, in the patrimonial character share those rights to be effecti$v e$, an attribute that is derived from the application of the theory of the fundamental rights of Robert Alexy, specifically from the perspective of the rights to something from the state as its object focus of this writing.

KEYWORDS: Constitution, rights and economic.

RECIBIDO: 01.12.2015

ACEPTADO: 09.12.2015 